\title{
Gyrokinetic electron acceleration in the force-free corona with anomalous resistivity
}

\author{
K. Arzner ${ }^{1}$ and L. Vlahos ${ }^{2}$ \\ 1 Paul Scherrer Institut, 5232 Villigen, Switzerland \\ e-mail: arzner@astro.phys.ethz.ch \\ 2 Department of Physics, Aristotle University of Thessaloniki, 54124 Thessaloniki, Greece \\ e-mail: vlahos@astro.auth.gr
}

Received 2 February 2006 / Accepted 27 March 2006

ABSTRACT

\begin{abstract}
Aims. We numerically explore electron acceleration and coronal heating by dissipative electric fields.
Methods. Electrons are traced in linear force-free magnetic fields extrapolated from SOHO/MDI magnetograms, endowed with anomalous resistivity $(\eta)$ in localized dissipation regions where the magnetic twist $\nabla \times \hat{\boldsymbol{b}}$ exceeds a given threshold. Associated with $\eta>0$ is a parallel electric field $\boldsymbol{E}=\eta \boldsymbol{j}$ that can accelerate runaway electrons. In order to gain observational predictions, we inject electrons inside the dissipation regions and follow them for several seconds in real time.

Results. Precipitating electrons that leave the simulation system at height $z=0$ are associated with hard X rays, and electrons that escape at height $z \sim 3 \times 10^{4} \mathrm{~km}$ are associated with normal-drifting type IIIs at the local plasma frequency. A third, trapped population is related to gyrosynchrotron emission. Time profiles and spectra of all three emissions are calculated, and their dependence on the geometric model parameters and on $\eta$ is explored. It is found that precipitation generally precedes escape by fractions of a second and that the electrons perform many visits to the dissipation regions before leaving the simulation system. The electrons impacting $z=0$ reach higher energies than the escaping ones, and non-Maxwellian tails are observed at energies above the largest potential drop across a single dissipation region. Impact maps at $z=0$ show the tendency of the electrons to arrive at the borders of sunspots of one polarity.

Conclusions. Although the magnetograms used here belong to non-flaring times, so that the simulations refer to nanoflares and "quiescent" coronal heating, it is conjectured that the same process, on a larger scale, is responsible for solar flares.
\end{abstract}

Key words. acceleration of particles - Sun: radio radiation - X-rays: gamma rays - methods: numerical

\section{Introduction}

Observations of radio waves and hard X-rays (HXR) from solar flares allow the study of the acceleration and propagation of high-energy electrons that are responsible for both types of emission. The picture emerging from the observations is generally complicated (Benz et al. 2005). While HXR (bremsstrahlung) emission is often associated with metric type-III radio groups (Aschwanden et al. 1995), the timing of individual type III's and the HXR fine structure is erratic.

Metric type III's are presumably caused by electron beams exciting Langmuir waves, which then couple to electromagnetic (observable) modes. Sometimes, there is perfect agreement between type III onsets and HXR maxima, but in other cases there is no obvious peak-to-peak correlation, or one of the emissions is absent altogether. As a trend, the type III's onsets were found to be delayed by fractions of a second against the HXR fine structures (Aschwanden et al. 1992; Arzner \& Benz 2005), although the type III frequency drift may assist such delays in cases where the type III onset could not be resolved properly. At millimeter and decimeter wavelengths, synchrotron emission (e.g., Giménez et al. 2005), decimetric radio continuum and decimetric pulsations (Saint-Hilaire \& Benz 2003), and decimetric spikes (Güdel et al. 1991) have also been found to be associated with HXR, roughly in the above decreasing order of association probability. These types of decimetric and millimetric radio emission are related to magnetically trapped electrons, where loss-cone instabilities are believed to be responsible for temporal structures (Kuijpers \& Slottje 1976; Aschwanden \& Benz 1988; Fleishman \& Melnikov 1998).

The variety of observed behaviour accounts, on the one hand, for the non-linear (coherent) radio emission processes. On the other hand, it reflects the geometric complexity of the active regions, which results from the nonlinear dynamics in the convection zone. In fact, the complex behaviour and the complex geometry are likely to be causes and effects of each other. Including a realistic amount of geometric complexity in a numerical model of solar flares was a major motivation for the present work.

Apart from the geometrical aspects, the modeling of solar flares requires the specification of a physical acceleration mechanism. As a working hypothesis, we assume here that acceleration is caused by DC parallel electric fields due to anomalous resistivity. Such fields are capable of accelerating high-energy electrons for which the electric force is no longer counterbalanced by the collisional drag (Dreicer 1960). It should be pointed out that runaway acceleration applies only to a small fraction of electrons in the high-energy tail of the electron distribution and that the majority of electrons is (Joule) heated rather than accelerated to superthermal energies. The electric fields envisaged above are of a macroscopic and dissipative nature and mark the irreversible release of non-potential magnetic energy. We thus 
follow a scenario proposed by Parker $(1972,1983,1993)$ where the random photospheric footpoint motion twists and shears the coronal magnetic loops so as to develop tangential discontinuities. This happens intermittently and in multiple localized regions throughout the solar corona and does not generally imply loop instability or global re-structuring of the solar corona. Parker's idea has initiated many investigations of coronal heating and eruptions (e.g., Schumacher \& Kliem 1996; Török \& Kliem 2001; Gudiksen \& Nordlund 2002) and has significantly improved the understanding of the global flaring process. In our view, a strong but heuristic argument for adopting dissipative (rather than conservative) electric fields as responsible for particle acceleration in solar flares is the intermittent and violent nature of flares, hinting at a catastrophic process that could not be cast in a Hamiltonian formalism. However, conservative electric fields, as used in most investigations of stochastic acceleration (e.g. Karimabadi et al. 1987; Schlickeiser 2003), may also act as particle accelerators.

In the present study, we adopt a test particle approach similar to the simulations of Matthaeus \& Lamkin (1986), Dmitruk et al. (2003, 2004), Arzner \& Vlahos (2004), and Arzner et al. (2006), but with two important modifications. First, the electromagnetic force fields are not taken from MHD turbulence simulations or random-phase turbulence proxies, but from observed magnetograms. Second, in order to span the many orders of magnitude between the electron Larmor radius and the size of magnetic loops, we allow for gyrokinetic motion, keeping track of the gyro phase in an approximate way. This technique enables us to follow the electrons over several seconds in real time (several $10^{8}$ gyro times), thereby reaching the time scales on which solar flares are observed. In the simulations, runaway electrons are injected at $t=0$ inside the localized dissipation regions and followed numerically along (and sometimes across) the magnetic field lines. Observational predictions for HXR and radio waves are obtained as the electrons impinge the chromosphere, get trapped, or escape to the higher corona.

The article is organized as follows. Section 2 describes the construction of the coronal field, Sect. 3 the particle orbits, Sect. 4 the numerical results and observational predictions. These are then summarised and discussed in Sect. 5.

\section{Coronal electromagnetic fields}

Our domain is a slab of height $H$, bounded at $z=0$ by the photosphere and filled with time-independent force-free (e.g., Gary 1989) magnetic fields. We do not ask for a perfect field reconstruction here, but for a generic configuration compatible with the observation. Accordingly, we use an elementary linear forcefree extrapolation from the normal photospheric magnetic field. This represents a local approximation at best (Wheatland 1999), and we have a slab thickness $H$ of not more than a few $10^{4} \mathrm{~km}$ in mind when fixing the physical scaling. (The linear force-free assumption predicts loops flaring with height, in contrast to the slender high-ranging loops observed by TRACE.) Further characteristic length and time scales are compiled in Table 1.

\subsection{Construction of the force-free magnetic field}

The force-free condition requires that $\nabla \times \boldsymbol{B}=\alpha \boldsymbol{B}$, where $\alpha$ is assumed to be constant ("linear" force-free field). Our construction of the force-free magnetic field follows the lines of Alissandrakis (1981), with modifications concerning the selection and number of Fourier modes. In order to avoid large but
Table 1. Notation and physical scaling.

\begin{tabular}{ccc}
\hline \hline Symbol & Meaning & Typical value \\
\hline$\Omega^{-1}$ & particle time unit & $10^{-9} \mathrm{~s}$ \\
$l_{0}$ & particle length unit & $0.3 \mathrm{~m}$ \\
$l$ & magnetogram resolution & $660 \mathrm{~km}$ \\
$L$ & magnetogram size & $1.7 \times 10^{5} \mathrm{~km}$ \\
$H$ & slab height & $3 \times 10^{4} \mathrm{~km}$ \\
$1 / \alpha$ & force-free scale & $2 \times 10^{4} \mathrm{~km}$ \\
$1 / u_{\mathrm{c}}$ & critical twist scale & $3 \times 10^{3} \mathrm{~km}$ \\
\hline
\end{tabular}

passive 3-dimensional arrays, the strategy is to locally compute $\boldsymbol{B}(\boldsymbol{x})$ from a restricted number of Fourier modes $\boldsymbol{b}(\boldsymbol{k}) \mathrm{e}^{\mathrm{i} \boldsymbol{k} \cdot \boldsymbol{x}}$. In terms of these, the force-free condition becomes i $\boldsymbol{k} \times \boldsymbol{b}(\boldsymbol{k})=$ $\alpha \boldsymbol{b}(\boldsymbol{k})$. This equation has only a non-trivial solution if $|\boldsymbol{k}|^{2}=\alpha^{2}$, which we write in the form

$k_{z}= \pm \sqrt{\alpha^{2}-k_{\perp}^{2}} \quad$ where $k_{\perp}^{2}=k_{x}^{2}-k_{y}^{2}$,

and if $\boldsymbol{b}(\boldsymbol{k})$ is proportional to

$\left(-k_{x} k_{z}+\mathrm{i} \alpha k_{y},-k_{y} k_{z}-\mathrm{i} \alpha k_{x}, k_{x}^{2}+k_{y}^{2}\right)$.

See MacLeod (1995) for a fuller discussion of the curl eigenfunctions. We assume $k_{x}$ and $k_{y}$ to be real, so that the magnetic field in the $(x, y)$-plane is bounded, while $k_{z}$ may be complex with a positive imaginary part. Thus modes decaying or oscillating with height are permitted, and we use a small admixture of oscillating modes to transport structures from the chromosphere to greater heights. From a physics point of view this procedure is justified by noticing that the outstreaming solar wind (in a 1-dimensional slab geometry) does not require $\boldsymbol{B}(z)$ to vanish as $z \rightarrow \infty$. Furthermore, different wave vectors are not rational multiples of each other, so that the magnetic field extends in a non-periodic, generic way across the $(x, y)$-plane. This irregular spacing of the Fourier components also avoids aliasing artifacts, and their small number allows a non-expensive evaluation of the magnetic field even if no fast Fourier transform is available. However, the restriction to just a few Fourier components makes an exact matching of the boundary conditions impossible. Instead, we require agreement only in a least square sense, and minimize the mean-square deviation of the sparse-Fourier field $B_{z}(x, y, 0)$ and the photospheric boundary field $B_{z 0}(x, y)$ in the square $L \times L$. Setting

$\boldsymbol{B}(\boldsymbol{x})=\operatorname{Re} \sum_{\boldsymbol{k}} C_{\boldsymbol{k}} \boldsymbol{b}(\boldsymbol{k}) \mathrm{e}^{\mathrm{i} \boldsymbol{k} \cdot \boldsymbol{x}}$,

normalizing the eigenvectors such that $\left|b_{z}\right|=1$, and using the (approximate) orthogonality of the harmonic functions over $L \times L$, the coefficients $C(\boldsymbol{k})$ are given by

$C(\boldsymbol{k}) \simeq \frac{\text { const. }}{N_{k}} \int_{L \times L} \mathrm{~d} x \mathrm{~d} y b_{z}^{*}(\boldsymbol{k}) \mathrm{e}^{-\mathrm{i} k_{x} x-\mathrm{i} k_{y} y} B_{z 0}(x, y)$.

The boundary field $B_{z 0}(x, y)$ is given on a square lattice (cell size $l^{2}$ ), and the integral (4) is computed numerically. "Complementary" solutions of the homogeneous boundary value problem (Chiu \& Hilton 1977; Petrie \& Lothian 2003) are disregarded. The sparse-Fourier wave vectors $\left(k_{x}, k_{y}\right)$ are selected by the following procedure. First, the fast Fourier transform of the discrete boundary field is computed and those $N_{k}$ (regularly spaced) wave vectors are chosen that have the highest power spectral density. Then, a random perturbation $\left(\Delta k_{x}, \Delta k_{y}\right) \lesssim \pi / l$ is added to break the exact periodicity. The eigenvectors (Eq. (2)) automatically ensure that $\nabla \cdot \boldsymbol{B}=0$. 

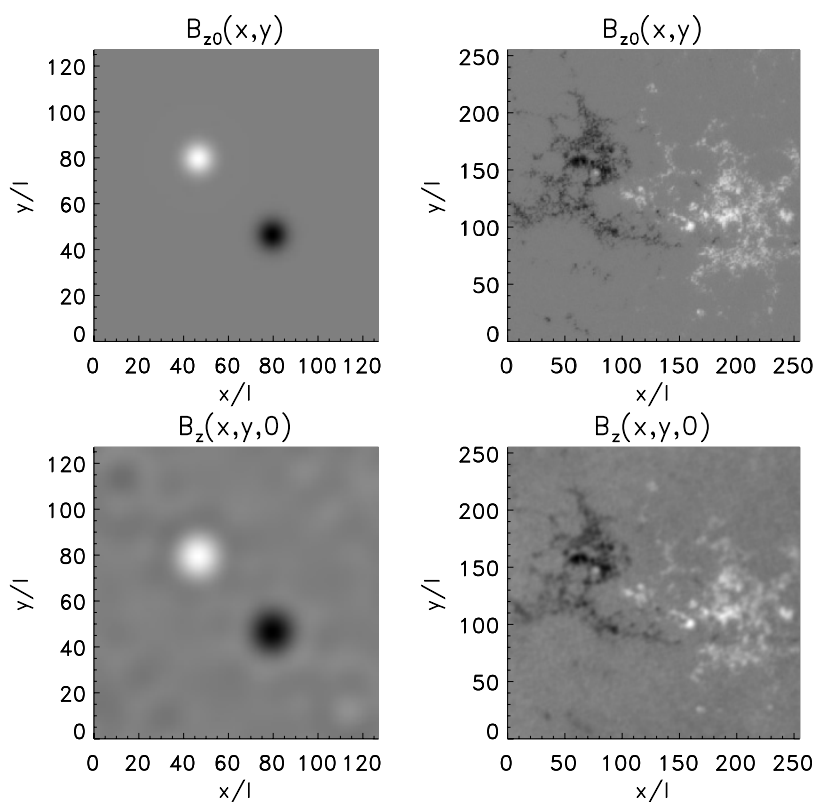

Fig. 1. Boundary fields (top) and their sparse-Fourier representations (bottom) of a bipolar configuration (left) and a SOHO/MDI magnetogram (right) recorded on August 17, 2002, 10:40 UT. The lower left corner of the magnetogram corresponds to $\left(78^{\prime \prime},-36^{\prime \prime}\right)$ in heliocentric coordinates, and the scale is $l=0.94^{\prime \prime}$.
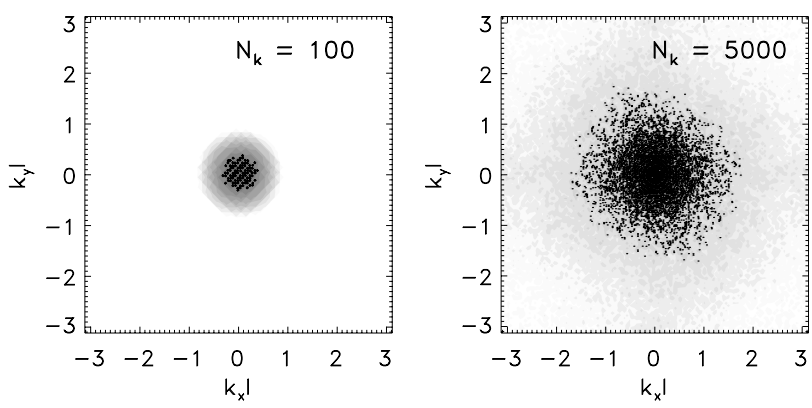

Fig. 2. Power spectral density (grayscale) of the boundary fields of Fig. 1 (top). Left: bipolar configuration; right: SOHO/MDI magnetogram. The sparse-Fourier components are marked by dots.

A test case with two Gaussian footpoints of opposite polarity is shown in Fig. 1 (left column), with the true boundary field $B_{z 0}(x, y)$ presented in the top left panel and its sparseFourier version $B_{z}(x, y, 0)$ presented in the bottom left panel. The sparse-Fourier version only contains $N_{k}=100$ components. This low number already provides a good approximation (correlation coefficient 0.94), because the Fourier spectrum (Fig. 2 left) is concentrated at the origin. When real data are used, a larger number of Fourier components is needed. This is illustrated in Fig. 1 (right column), showing a SOHO/MDI magnetogram (top right) and its sparse-Fourier representation (bottom right) using 5000 Fourier components (Fig. 2 right). The magnetogram is located close to the centre of the solar disc, so that projection effects are negligible. The magnetogram resolution is $l=660 \mathrm{~km}$ (rescaled from the original SOHO/MDI resolution), and the correlation coefficient between the magnetogram and its sparse-Fourier representation is 0.93 . The magnetogram belongs to a non-flaring configuration and therefore the force-free extrapolation should be a reasonable approximation. Although the smallest scales are not well represented by the sparse-Fourier approximation, they rapidly decay with height (Eq. (1)), so that the

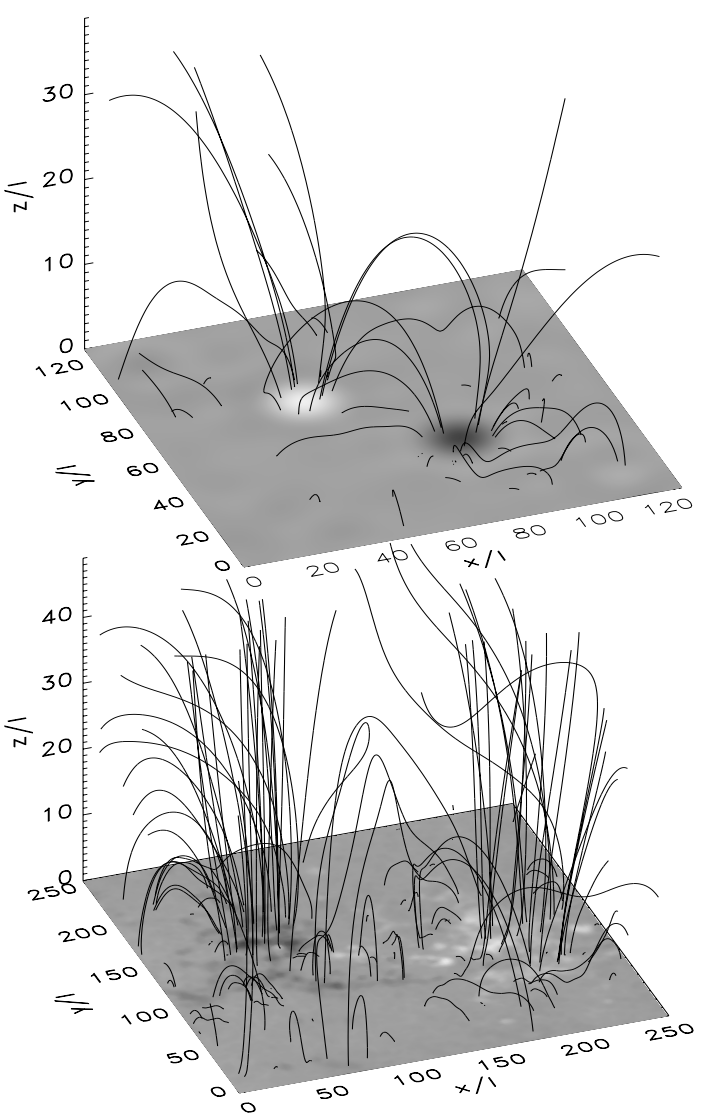

Fig. 3. Sparse-Fourier force-free magnetic field lines extrapolated from bipolar (top) and magnetogram (bottom) boundary data.

approximation becomes better as $z$ increases. This fact is used to save computation time by restricting the field computation to components with $\left|\mathrm{e}^{\mathrm{i} k_{z} z}\right|>10^{-3}$.

\subsection{Properties of the magnetic field}

Figure 3 displays the force-free magnetic fieldlines of the bipolar test case (top) and the SOHO/MDI magnetogram (bottom). The $\alpha$ parameter is such that $\alpha l=0.03$, corresponding to a force-free scale $1 / \alpha$ of about $2 \times 10^{4} \mathrm{~km}$. The field lines start at $z=0$ at random with density proportional to $|\boldsymbol{B}|$. Several field-line integration schemes have been tested, and the force-free condition was also verified using finite 3-dimensional differences. Most field lines connect the two poles of opposite polarity, but not all, because the sparse-Fourier field does not vanish outside the poles, nor outside $L \times L$.

The absolute scaling of $\boldsymbol{B}$ is constrained by the SOHO/MDI data for Fig. 3 (bottom), which give a longitudinal photospheric rms field $\sqrt{\left\langle B_{z 0}(x, y)^{2}\right\rangle}=50 \mathrm{G}$ with excursions to $\pm 400 \mathrm{G}$ at the footpoints. These values are typical of active regions; the average over the quiet solar surface is lower $(\sim 1 \mathrm{G}$, see Kotov 2002). The mean-square magnetic field strength is related to the Fourier amplitudes by $\left\langle B_{x}(x, y, 0)^{2}\right\rangle=\frac{1}{2} \sum_{k}\left|b_{x}(\boldsymbol{k})\right|^{2}$ and similar relations for $B_{y}$ and $B_{z}$.

\subsection{Electric field}

Since the configuration is assumed to be time-independent, the electric field has only an Ohmic component $\boldsymbol{E}=\eta \boldsymbol{j}$. The (scalar) resistivity $\eta$ is either zero or anomalous, i.e., much higher than 

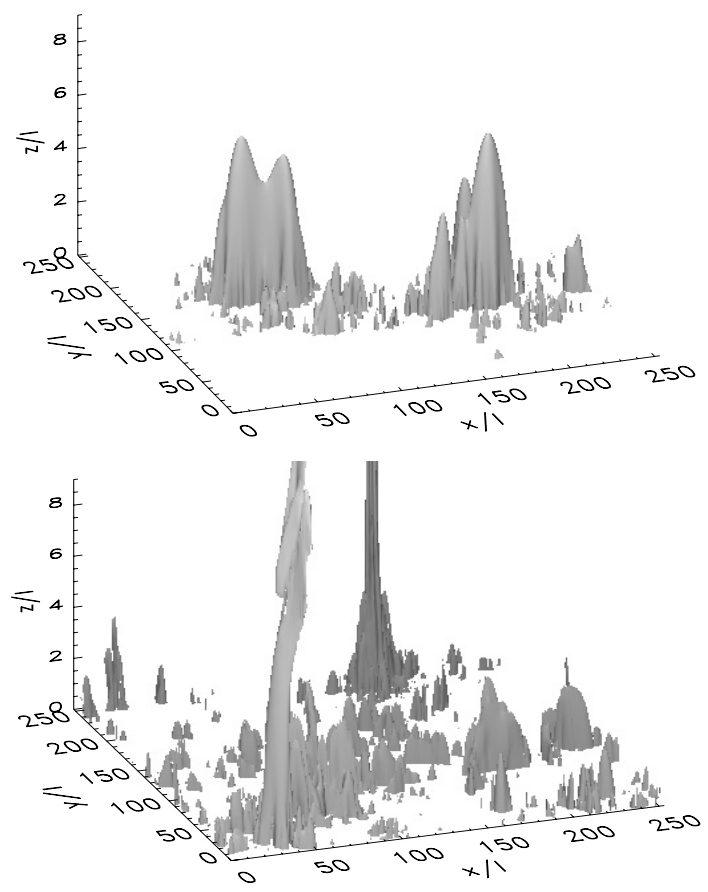

Fig. 4. Different definitions of dissipation regions. Top: current threshold. Bottom: magnetic twist threshold. Both panels refer to the magnetogram of Fig. 3 (bottom). It is the twist threshold which is used in the sequel of this paper.

the collisional value $\eta_{\text {Spitzer }}$ (Helander 2002). Regions with $\eta \gg$ $\eta_{\text {Spitzer }}$ are called "dissipation regions" here. Owing to the forcefree condition $\nabla \times \boldsymbol{B}=\alpha \boldsymbol{B}$, the electric field $\boldsymbol{E}=\eta \alpha \boldsymbol{B}$ is purely parallel.

Different criteria for the occurrence of anomalous resistivity have been proposed, relying on linear micro-instabilities or heuristic arguments. A well-known argument limits the electric current to $j_{\mathrm{c}}=e n c_{\mathrm{s}}$ where $c_{\mathrm{s}}$ is the speed of sound (Papadopoulos 1980); an excess $|\nabla \times \boldsymbol{B}|>j_{\mathrm{c}}$ is then said to produce shocks and dissipation in the current-carrying electron fluid. The resulting dissipation region $|j|>j_{\mathrm{c}}$ is depicted in Fig. 4 (top) for the configuration of Fig. 3 (bottom). As the forcefree current is proportional to the magnetic field, the dissipation regions simply delineate the magnetic field strength.

Another approach invokes the twist or shear of magnetic field lines rather than the electric current density. The physical motivation for this stems from the observation that regions of larger magnetic field should also be able to guide stronger currents before disruption. Thus the automatic increase of $j_{\mathrm{c}}$ with increasing $|\boldsymbol{B}|$ should be compensated, which is achieved by considering the quantity

$\hat{b}=\frac{B}{|B|}$

rather than $\boldsymbol{B}$. The criterion for the occurrence of anomalous resistivity is then defined by

$|\nabla \times \hat{\boldsymbol{b}}|<u_{\mathrm{c}}$.

The resulting dissipation regions are shown in Fig. 4 (bottom); the top and bottom panels represent equal dissipation volumes.

It follows from the force-free condition that $\hat{\boldsymbol{b}} \cdot \nabla \times \hat{\boldsymbol{b}}=\alpha$. Thus, for constant $\alpha$, the field-aligned component of the magnetic twist $\nabla \times \hat{\boldsymbol{b}}$ is constant throughout the whole volume. This implies, in particular, that $|\nabla \times \hat{\boldsymbol{b}}|$ can never be smaller than $\alpha$, and that the threshold $u_{\mathrm{c}}$ must be higher than $\alpha$. In Fig. 4 (bottom), $u_{\mathrm{c}} / \alpha=6.6$. Since small scales with $k_{\perp}>\alpha$ decay exponentially with height, the steep gradients, and thus the dissipation regions, tend to accumulate at low altitudes. This agrees with the observation that the coronal heating function is localized within a height range $\$ 10^{4} \mathrm{~km}$ (Aschwanden 2001). The dissipation regions are thus not uniformly distributed in height, and the dissipative volume $V_{\mathrm{d}}$ can be characterised by a column height

$h_{\mathrm{d}}=V_{\mathrm{d}} / L^{2}$

rather than by a volume fraction referring to the full numerical domain $L^{2} H$. In the case of Fig. 4 one has $h_{\mathrm{d}}=0.19 l$.

Condition (6) is local. Non-local versions of Eq. (6) have been used by Georgoulis \& Vlahos (1998) along pairs of magnetic field lines and by Vlahos \& Georgoulis (2004) on a discrete lattice. We use Eq. (6) here, since it can be evaluated at the particle position and thus simplifies the simulation. The threshold $u_{\mathrm{c}}$ is chosen such that $u_{\mathrm{c}} l=0.2$.

\section{Particle orbits}

\subsection{Dimensionless units and physical scaling}

The system of units used in this article is similar to the one in Arzner \& Vlahos (2004). Time is measured in units of $\Omega^{-1}$ where $\Omega^{2}=\frac{1}{2} \sum_{\boldsymbol{k}}|\boldsymbol{b}(\boldsymbol{k})|^{2}$ is the non-relativistic mean-square gyro frequency at $z=0$. Velocity is measured in units of the speed of light, energy in units of $m c^{2}$, momentum in units of $m c$, and distance in units of $l_{0}=c \Omega^{-1}$. We take $\left\langle|\boldsymbol{B}(x, y, 0)|^{2}\right\rangle^{1 / 2}=50 \mathrm{G}$ (see Sect. 2.2) to fix the absolute scaling, so that the electron cyclotron frequency is $140 \mathrm{MHz}$, the time unit is $\Omega^{-1}=10^{-9} \mathrm{~s}$, and the length unit $l_{0}=0.3 \mathrm{~m}$. The electric field is measured in units of $c B$, and the resistivity $\eta$ in units of $c^{2} \Omega^{-1}$.

\subsection{Exact orbits}

In the above unit system, the exact particle equations of motion read

$$
\begin{aligned}
\frac{\mathrm{d} \boldsymbol{x}}{\mathrm{d} t} & =\boldsymbol{v} \\
\gamma \frac{\mathrm{d} \boldsymbol{v}}{\mathrm{d} t} & =\boldsymbol{v} \times \boldsymbol{B}+\boldsymbol{E}-(\boldsymbol{v} \cdot \boldsymbol{E}) \boldsymbol{v}
\end{aligned}
$$

where $\boldsymbol{E}=\eta \nabla \times \boldsymbol{B}$ and $\gamma=1 / \sqrt{1-v^{2}}$ is the Lorentz factor. Equations (8) and (9) are integrated by a traditional CashKarp/Runge-Kutta method (Press et al. 1998) with an adaptive time step. The sparse-Fourier fields are computed at a lower rate, together with their Jacobians, from which a linear extrapolation to the actual particle position is made. The time step is chosen such that both the Cash-Karp error and the field extrapolation error remain within given bounds (Appendix A).

\subsection{Gyrokinetic approximation}

In the magnetic fields considered here, ranging from a few to a few hundred Gauss, and for typical kinetic energies between $1 \mathrm{keV}$ and $1 \mathrm{MeV}$, the electron Larmor radius $\gamma v_{\perp} / \Omega$ varies from centimetres to hundreds of metres, and is therefore small compared to the resolution of the magnetic field $\left(l \sim 10^{3} \mathrm{~km}\right)$. The motion is thus almost always adiabatic, with possible exceptions at critical points in the magnetic field, and inside the dissipation regions. This suggests a gyrokinetic approximation with the possibility of switching to exact orbits if necessary. 
We base our guiding-centre mover on a relativistic (Brizard 1999) version of the gyrokinetic equations of Littlejohn (1981, 1983). The variables considered are $\left(\boldsymbol{X}, p_{\|}, \Theta, \mu\right)$, where $\boldsymbol{X}$ is the guiding centre position, $p_{\|}=\gamma v_{\|}$the momentum parallel to the magnetic field, $\Theta$ the gyro phase, and $\mu=\frac{1}{2} \gamma^{2} v_{\perp}^{2} / B$ is the magnetic moment, which is a motion integral in the present approximation. The coordinates $\left(\boldsymbol{X}, p_{\|}\right)$evolve according to

$$
\begin{aligned}
\frac{\mathrm{d} \boldsymbol{X}}{\mathrm{d} t} & =\frac{1}{B_{\|}^{*}}\left(\frac{p_{\|}}{\gamma} \boldsymbol{B}^{*}+\frac{\mu}{\gamma} \hat{\boldsymbol{b}} \times \nabla B-\hat{\boldsymbol{b}} \times \boldsymbol{E}^{*}\right) \\
\frac{\mathrm{d} p_{\|}}{\mathrm{d} t} & =-\frac{1}{B_{\|}^{*}} \boldsymbol{B}^{*} \cdot\left(\frac{\mu}{\gamma} \nabla B-\boldsymbol{E}^{*}\right)
\end{aligned}
$$

where $\gamma=\sqrt{1+p_{\|}^{2}+2 \mu B}$ and

$\boldsymbol{B}^{*}=\boldsymbol{B}+p_{\|} \nabla \times \hat{\boldsymbol{b}}$

$\boldsymbol{E}^{*}=\boldsymbol{E}-p_{\|} \frac{\partial \hat{\boldsymbol{b}}}{\partial t}$

with $B_{\|}^{*}=\boldsymbol{B}^{*} \cdot \hat{\boldsymbol{b}}$. For our time-independent force-free magnetic field, $\boldsymbol{E}^{*}$ equals $\boldsymbol{E}$ and the last term in Eq. (10) vanishes.

In order to switch back to exact orbit integration, we need to keep track of the gyro phase. In gyrokinetic approximations, the gyro phase is not unambiguously defined but subject to gauge freedom. Accordingly, various definitions have been proposed. We follow Littlejohn (1988) here. Let $\hat{\boldsymbol{e}}$ be a unit vector in the direction of the initial $(t=0)$ Larmor radius. The vector $\hat{\boldsymbol{e}}_{1}$ is perpendicular to the direction $\hat{\boldsymbol{b}}$ of the local magnetic field, and a local Cartesian triad is completed by setting $\hat{\boldsymbol{e}}_{2}=\hat{\boldsymbol{e}}_{1} \times \hat{\boldsymbol{b}}$. The task is to follow the unit vector $\hat{\boldsymbol{e}}_{1}$ along the gyro centre, introducing a minimum of twist. This is achieved by solving the parallel transport (Fermi-Walker type) equation

$\frac{\mathrm{d} \hat{\boldsymbol{e}}_{1}}{\mathrm{~d} s}=-\hat{\boldsymbol{b}}\left(\frac{\mathrm{d} \hat{\boldsymbol{b}}}{\mathrm{d} s} \cdot \hat{\boldsymbol{e}}_{1}\right)$

where $s$ is the distance along the gyrocentre orbit. The gyro phase, with respect to the direction $\hat{\boldsymbol{e}}_{1}$, is then given by $\Theta=$ $\int_{0}^{t} \gamma^{-1}\left|\boldsymbol{B}\left(\boldsymbol{X}\left(t^{\prime}\right)\right)\right| \mathrm{d} t^{\prime}$, and the exact orbit parameters $(\boldsymbol{x}, \boldsymbol{v})$ can be retrieved from $\left(\boldsymbol{X}, v_{\|}, \mu, \Theta, \hat{\boldsymbol{e}}_{1}\right)$. More details and the benchmarking of our numerical approach are described in Appendix A.

\section{Simulation results and observational predictions}

The slab-shaped simulation domain suggests a simple scheme of observational diagnostics, which is sketched in Fig. 5. In this scheme, HXRs are associated with electrons impacting $z=0$, where they are stopped by the rapidly increasing density ${ }^{1}$. Electrons that remain magnetically trapped are associated with gyrosynchrotron emission, and electrons that leave the simulation domain at $z=H$ are associated with radio type III emission at the local plasma frequency.

We performed several simulation runs with varying parameters of the electromagnetic fields. One of these runs is discussed in detail in Sects. 4.1 to 4.4, while the outcome of the others is summarised in Sect. 4.5. In all simulations, electrons with initial velocity $v_{0}=0.1 c\left(E_{0}=2.6 \mathrm{keV}\right)$ are injected at $t=0$

${ }^{1}$ Strictly speaking, $z=0$ delineates the photosphere probed by the SOHO/MDI magnetogram, and not the density step (transition region) seen by the precipitating electrons. We do not make this distinction here since our model does not include a background density profile.

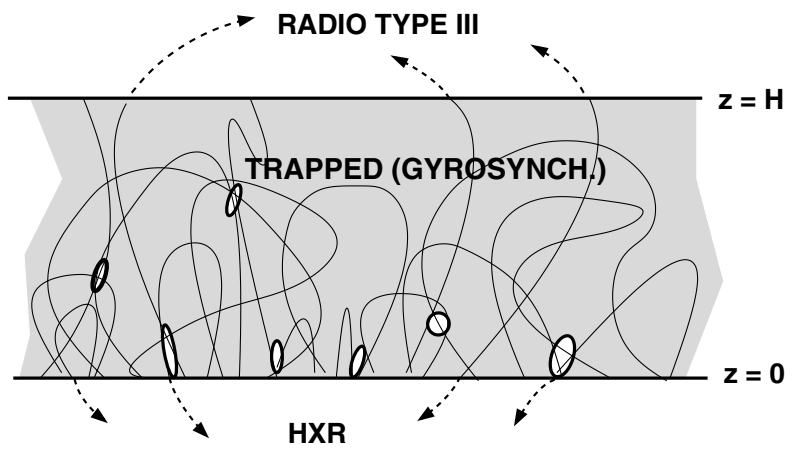

Fig. 5. Schematic allocation of different emissions from high-energy electrons. Precipitating electrons are associated with HXRs, and escaping and trapped electrons with radio emission. Black lines symbolize magnetic field lines; boldface ellipses symbolize dissipation regions.

inside the dissipation regions, and traced until one of the following conditions is met: $z<0$ ("precipitation"), $z>H$ ("escape"), or $t>T_{\max }$ ("trapping"). The simulation duration $T_{\max }$ is long compared to the average arrival times at $z=0$ and $z=H$. Also, $T_{\text {max }}$ is long compared to the free escape time $\tau_{0} \doteq H / v_{0}$ calculated on grounds of the initial velocity. The synchronized injection at $t=0$ is somewhat artificial since we used magnetograms of a non-flaring active region. We chose this initial condition in order to model HXR and radio transients and thus provide additional (timing) diagnostics in situations where an observable flare pulse occurs, based on the assumption that flares are largescale versions of the process considered here. The predictions will, though, only be qualitative, since the time evolution of the flaring region is not taken into account.

\subsection{Electrons}

The outcome of a typical simulation is summarised in Fig. 6. The top panel represents the exit rates at $z=0$ and $z=H$. Most particles exit through the $z=0$ boundary, and the precipitation rate has a sharp peak at $\Omega t \sim 10^{7}$ (not resolved in Fig. 6). The escape rate peaks later. The remaining (trapped) population gradually decreases (Fig. 6 middle; smaller times than the free escape time $\Omega \tau_{0}=6 \times 10^{8}$ are not shown). A detailed look at the particle orbits shows that these usually perform numerous visits to the dissipation regions before reaching relativistic energies. The fraction of time spent by the electrons inside the dissipation regions is quite different for the different populations. While precipitating particles spend about $10 \%$ of their time inside the dissipation regions, the escaping ones do so only for about $2 \%$. The trapped ones spend $14 \%$ of their time inside the dissipation regions, but do not systematically gain energy. In contrast, the escaping and - more pronounced - precipitating particles gain relativistic energies. The terminal energy spectra are shown in Fig. 6 (bottom). Non-Maxwellian tails occur in the precipitating spectrum at energies $E / m c^{2} \sim 1$. The trapped electron spectrum refers to the end of the simulation $\left(t=T_{\max }\right)$.

\subsection{Hard $X$ rays}

Light curve and spectrum. In our model, the precipitating population is associated with HXR bremsstrahlung, with the HXR time profile directly proportional to the electron exit time density at $z=0$ (Fig. 7). Since $z=0$ is an absorbing boundary, we use the thick-target approximation. The (orientationaveraged) bremsstrahlung cross section is taken from the fully 

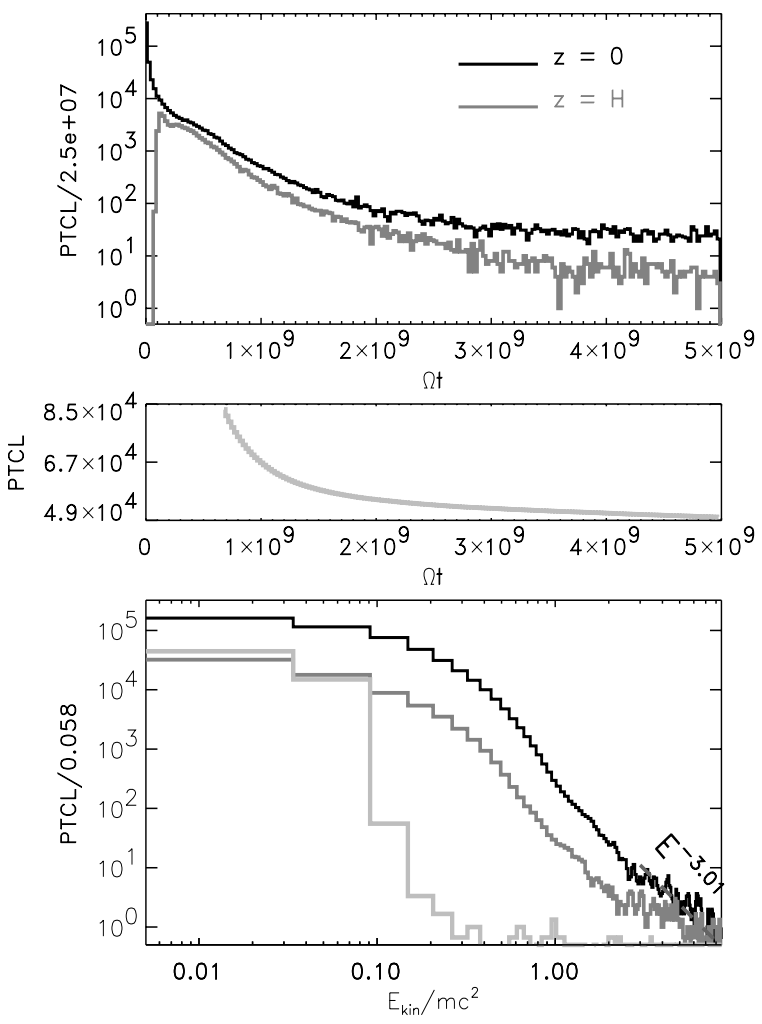

Fig. 6. Precipitating, escaping, and trapped electrons. Top: exit rates at the slab boundaries. Middle: remaining (trapped) particles. Bottom: terminal kinetic energies. $\Omega t=5 \times 10^{9}$ corresponds to $5 \mathrm{~s}$ in real time.

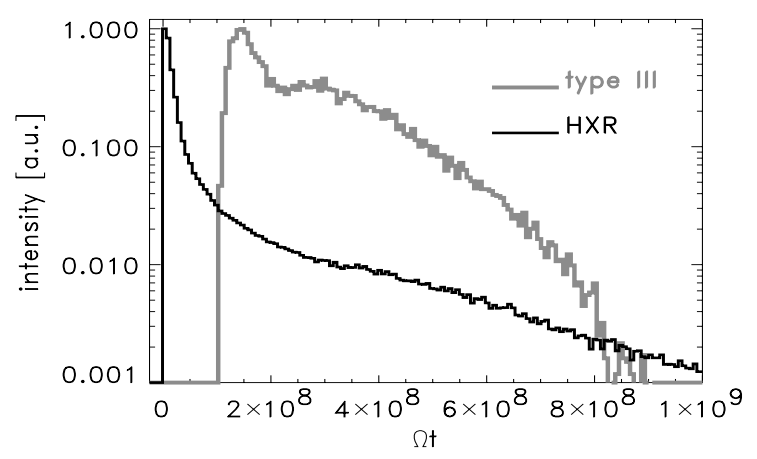

Fig. 7. Simulated HXR and type III light curves.

relativistic formula 3BN of Koch \& Motz (1959). The resulting HXR spectrum is shown in Fig. 8. At energies up to $\sim 40 \mathrm{keV}$, it can be fitted by a (hard) power law with index 1.4 (Fig. 8 inlet). At higher energies, it decays exponentially and finally drops off faster than exponentially at $\sim 800 \mathrm{keV}$. Most of the exponential part and the super-exponential decay could usually not be observed with X-ray observatories like RHESSI because of the limited dynamic range (2-3 orders of magnitude for $\mathrm{M}$ class flares; see Grigis \& Benz 2004).

Impact map. The present simulation also predicts the sites at which electrons impinge on the chromosphere (Fig. 9 left), and allows a comparison of this with the boundary magnetic field (Fig. 9 right, repeated here from Fig. 1 for more clarity). Since we inject particles into all dissipation regions simultaneously, the impact map represents a statistical prediction. There are two observations to be made: first, the electrons tend to precipitate at negative magnetic polarity. Second, the impact density peaks

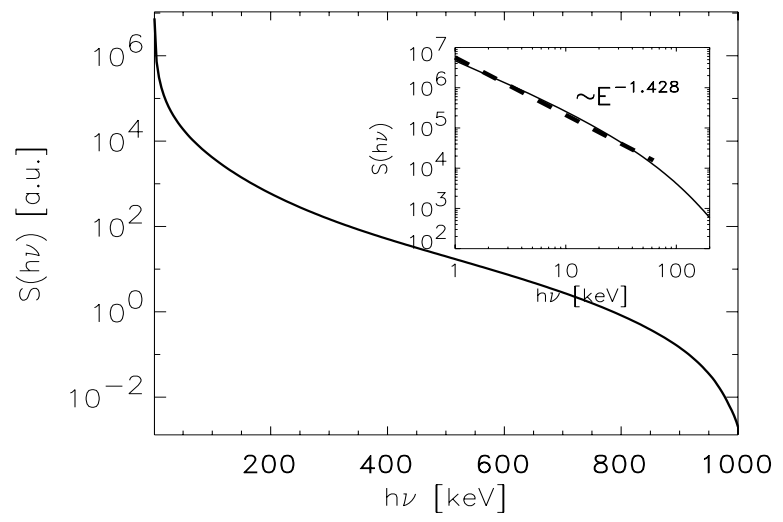

Fig. 8. Thick-target bremsstrahlung spectrum from the precipitating electrons.
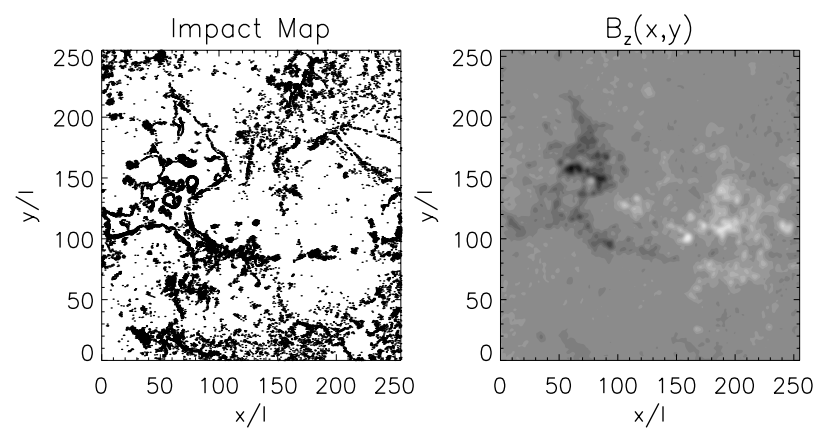

Fig. 9. Electron precipitation sites (left) and boundary magnetic field (right). Positive magnetic polarity is light; negative polarity is dark.

not at the centre of the sunspots or filaments, but at their borders. The first observation is a simple consequence of the constant- $\alpha$ assumption, by which the electric acceleration is always parallel or antiparallel to the magnetic field ${ }^{2}$. The second observation is a consequence of the mirroring in converging magnetic field lines, which makes it hard for electrons to penetrate down to $z=0$ in regions of maximal $|\boldsymbol{B}(x, y, 0)|$. The actual impact map represents a trade-off between the number of downstreaming electrons and their reflection probability, which is optimal at the sunspot/filament boundaries.

\subsection{Electron beams}

Electrons that escape to altitudes $z>H$ are considered as a proxy for type III radio bursts. Contrary to the incoherent bremsstrahlung and gyrosynchrotron radiation, the plasma emission of type III bursts is coherent and therefore not proportional to the number of emitting electrons. The non-linear dependence (say, $\propto N^{\xi}$ with $\xi>1$ ) compensates for the relatively tenuous escaping population. The resulting time signal is shown in Fig. 7, together with the HXR light curve. The scaling of the two intensities is arbitrary. As the background plasma density is not specified in our model, we cannot predict the emission frequency of the plasma waves. However, we may consult the observed density profiles at height $H \sim 3 \times 10^{4} \mathrm{~km}$, yielding $n_{\mathrm{e}} \sim 2.5 \times 10^{8} \mathrm{~cm}^{-3}$ averaged over the quiet corona (Fludra 1999). This number varies by a factor 3 when equator-to-pole variation is taken into account (Gallagher et al. 1999), and may be larger by a factor 10 above faculae (Dumont et al. 1982). Thus

\footnotetext{
${ }^{2}$ We have assumed positive $\alpha$ here and that the electrons have a positive charge.
} 


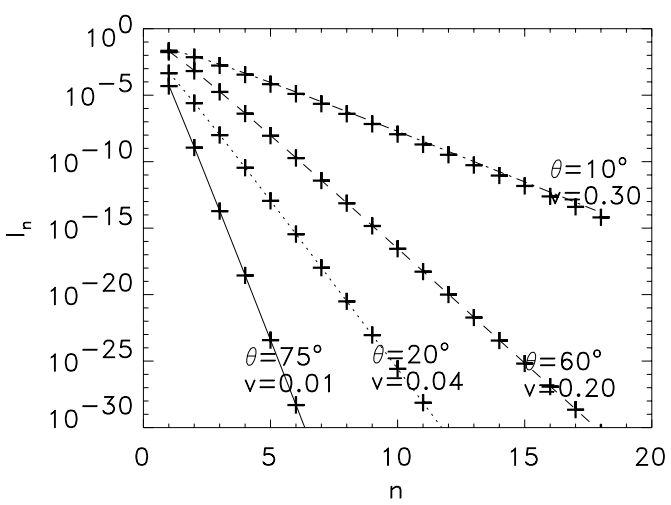

Fig. 10. Gyrosynchrotron intensity according to the exact expression (Eq. (15) - crosses) and its approximation (Eq. (16) - dotted line).

we associate a plasma density of $10^{8} \mathrm{~cm}^{-3}$ to $2 \times 10^{9} \mathrm{~cm}^{-3}$ with the $z=H$ boundary of our simulation slab, corresponding to a plasma frequency of 100 to $400 \mathrm{MHz}$.

\subsection{Gyrosynchrotron emission}

The electrons trapped in closed loops are thought to be responsible for gyrosynchrotron radiation, manifesting in microwave solar continuum bursts ${ }^{3}$. In this case there are many bursts that are optically thin at all frequencies (Fleishman et al. 2003), and we assume optically thin radiation for simplicity. Moreover, we neglect the plasma response and consider emission in a vacuum. Therefore, our results apply to the emission at frequencies above the spectral peak provided by either optical thickness or the Razin effect. An electron on a circular orbit emits then, at frequency $n \omega / \gamma$, the intensity (Schott 1912)

$I_{n}=\frac{n^{2} \omega^{2}}{\gamma^{2}}\left(\tan ^{2} \theta J_{n}^{2}(n v \cos \theta)+v^{2} J_{n}^{\prime 2}(n v \cos \theta)\right)$

where $n$ is an integer, $\omega=e B / m$ the local cyclotron frequency, and $\theta$ the angle between the gyration plane and the line of sight that is taken along the $z$-direction. In our simulation, most trapped electrons have velocities $v \ll 1$ (Fig. 6 bottom), so that the argument of the Bessel functions in Eq. (15) is small and $J_{n}(z)$ can be approximated by $(2 \pi n)^{-1 / 2}(e z / 2)^{n}$ (Abramowitz \& Stegun 1970), yielding

$I_{n} \simeq \frac{\omega^{2}}{\gamma^{2}} \frac{1+\sin ^{2} \theta}{\cos ^{2} \theta} \frac{n}{2 \pi}\left|\frac{e v \cos \theta}{2}\right|^{2 n}$,

where Euler's number $e=2.718$ is not to be confused with the elementary charge. The relative accuracy of the approximation (16) is about $|v \cos \theta|$ for all $n$; a numerical illustration is shown in Fig. 10. From Eq. (16) we see that the intensity $I_{n}$ decays exponentially with $n$, so that the radiation is concentrated at low harmonics. In the limits $v \rightarrow 0$ and $\theta \rightarrow 90^{\circ}$, only the fundamental $(n=1)$ contributes. If the velocity has a component parallel to the magnetic field and to the observing direction, this results in a Doppler shift $\omega \rightarrow \omega^{*}$, and $v$ in Eqs. (15), (16) has to be replaced by $v_{\perp}$, the component perpendicular to the magnetic field. Neglecting dispersion and absorption, we may thus obtain the radio spectrum by accumulating a histogram of the frequencies $n \omega^{*} / \gamma$ with weight $I_{n}$ for all particles and times. The result is shown in Fig. 11. Note that the spectrum decays monotonically and approximately exponentially with frequency, which

3 In contrast, narrowband decimetric continua are explained better by transition radiation (Fleishman et al. 2005; Nita et al. 2005).

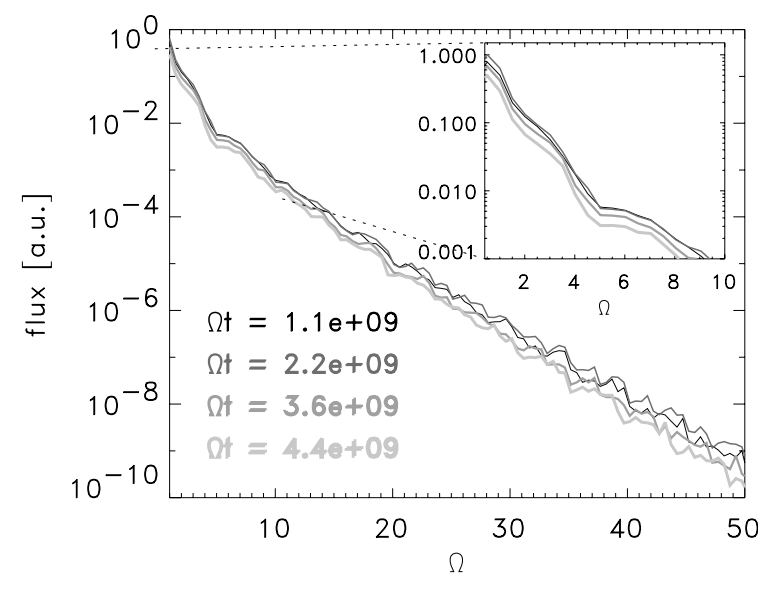

Fig. 11. Optically thin gyrosynchrotron spectrum emitted in the $z$ direction. Different curves represent different times. $\Omega=1$ corresponds to about $1 \mathrm{GHz}$.

Table 2. Simulation parameters. In all simulations, $\Omega^{-1}=10^{-9} \mathrm{~s}$ and $u_{\mathrm{c}} l=0.2$.

\begin{tabular}{cccccccc}
\hline \hline run & $N_{k}$ & $N_{x}$ & $l[\mathrm{~km}]$ & $H / l$ & $\alpha l$ & $\eta\left[\mathrm{c}^{2} / \Omega\right]$ & $h_{\mathrm{d}} / l$ \\
\hline 1) & 5000 & 128 & 875 & 40 & 0.05 & 4.38 & 0.56 \\
2) & 5000 & 256 & 660 & 50 & 0.05 & 6.84 & 0.50 \\
$3)$ & 5000 & 256 & 660 & 50 & 0.03 & 6.84 & 0.19 \\
$4)$ & 5000 & 256 & 660 & 64 & 0.01 & 6.84 & 0.13 \\
$5)$ & 1000 & 256 & 660 & 64 & 0.01 & 6.84 & 0.05 \\
$6)$ & 1000 & 256 & 660 & 64 & 0.01 & 1.37 & 0.08 \\
\hline
\end{tabular}

is different from the familiar synchrotron $(\gamma \gg 1)$ shape with powerlaw rise and decay. Here, the exponential decay is mostly caused by the harmonics dependence (Eq. (16)) but also supported by the exponential decay of magnetic field with height. Individual harmonics are washed out because of the magnetic field inhomogeneity.

\subsection{Parameter exploration}

We performed various production runs, involving a total CPU time of about 4 months. Different runs have different values for the parameters $H$ (slab height), $\alpha$ (force-free parameter), and $\eta$; see Table 2. The critical twist is fixed at $u_{\mathrm{c}} l=0.2$. The simulation discussed in Sects. $4.1-4.4$ is labeled 3 and represents an intermediate case. Each simulation involves several $10^{5}$ particles. An overview on the simulation results is presented in Fig. 12. Column a) contains the electron exit rates at $z=0$ (black line) and $z=H$ (gray line) during the first third of the simulation time, normalized by the total number of simulated electrons. Column b) shows the energy distributions at the end of the simulation $(t=$ exit time, or $\left.t=T_{\max }\right)$. Translation of the electron results into HXR and radio wave predictions proceeds similar as in Sects. 4.2 to 4.4 , and preserves the hardness ordering of the spectra.

The simulations 1 to 6 may be summarised by saying that the results of Sects. 4.1 to 4.4 are generic, in the sense that the general shape and relative timing of the light curves are robust against variation in $(H, \alpha, \eta)$. However, the time scaling, the division into precipitating and escaping populations, and the spectrum depend on the parameters (Table 2). The time delay between the precipitation pulse and the onset of escape (Fig. 12 left) increases naturally with slab height $H$. It also depends on the force-free parameter $\alpha$, yet in a subtler way. From the comparison of simulations 2 and 3, it is seen that a higher $\alpha$ favours 

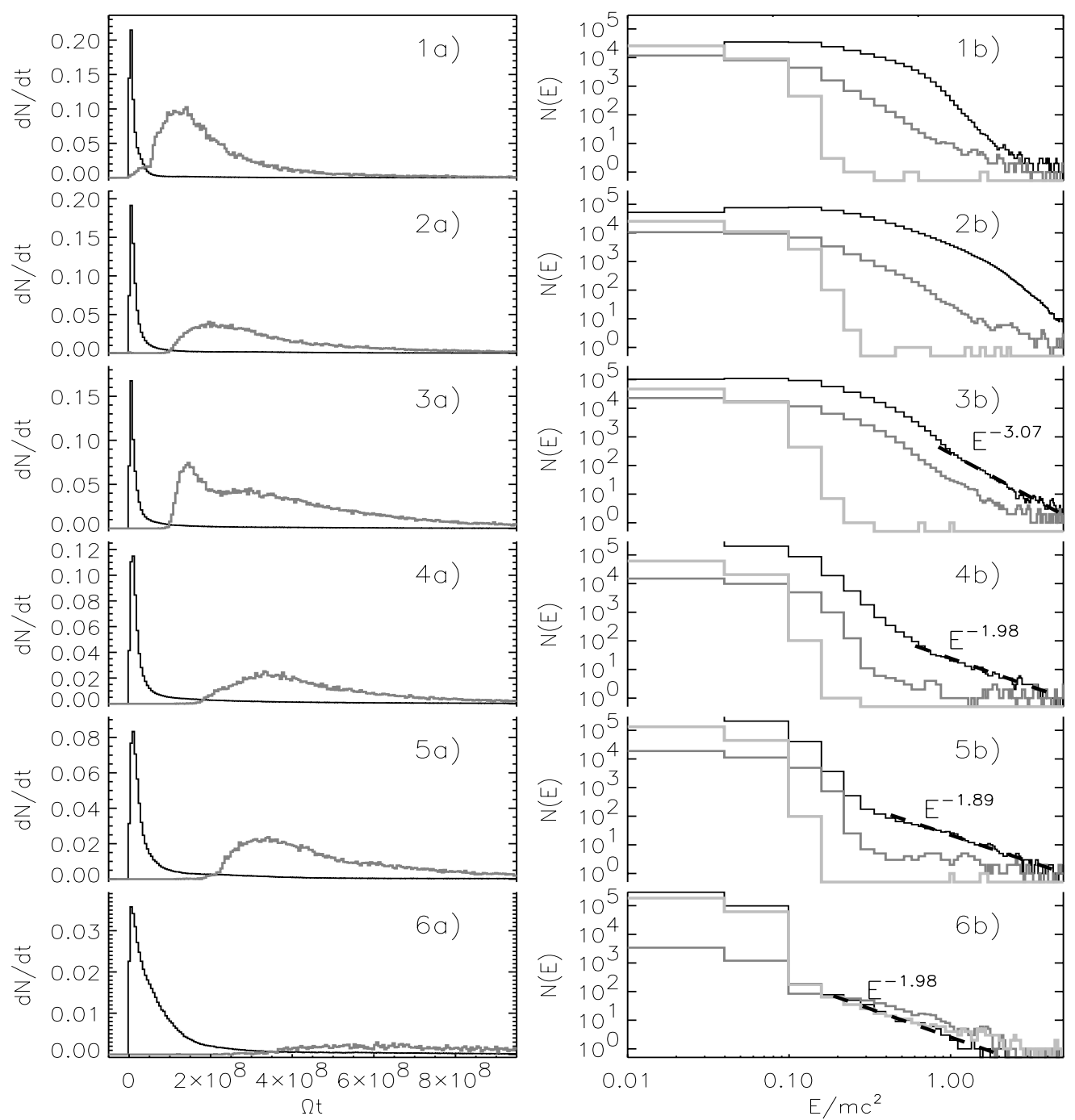

Fig. 12. Parameter exploration. Left column: exit rates. Right column: terminal energy spectra. Black (gray, light gray) lines refers to precipitating (escaping, trapped) populations. See Table 2 for the simulation parameters.

prompt escape. This is not immediately obvious, since a higher $\alpha$ implies a smaller force-free scale $1 / \alpha$ and thus smaller magnetic loops that do not protrude to $z=H$. However, a higher $\alpha$ also admits more non-decaying modes $\left(k_{\perp}<\alpha\right)$ and thus more open field lines along which the electrons can escape. Moreover, a higher $\alpha$ implies larger curvature, so that the criticality condition (Eq. (6)) is more frequently met and the accelerating volume is larger $\left(h_{\mathrm{d}}=0.5 l\right.$ in simulation 2 but $h_{\mathrm{d}}=0.19 l$ in simulation 3). As a net effect, a large force-free parameter $\alpha$ thus favours rapid acceleration and escape. A similar enhancement of acceleration by the presence of small scales accounts for the difference between panels 4 and 5 in Fig. 12. These simulations differ only by the number of Fourier components. Run 4 contains wave vectors up to $k_{\perp} l \leq 1.94$, whereas run 5 contains wavevectors $k_{\perp} l \leq 0.8$. Accordingly, run 4 reaches somewhat higher energies (panels $4 \mathrm{~b}$ and $5 \mathrm{~b}$ ). The escape time also increases (and the escape probability decreases) with decreasing anomalous resistivity and thus with decreasing electric field (5a vs. 6a). The trapped component is throughout softest, except for the case of very small resistivity (run 6) where all 3 populations behave similarly for $E / m c^{2} \gtrsim 0.1$. Run 6 is similar to run 5 but with smaller resistivity, balanced by a somewhat larger dissipation volume.

As a general trend, Fig. 12 (top to bottom) shows that smaller dissipation volumes and weaker electric fields yield a more gradual evolution and less intermediate energies $\left(0.1<E / m c^{2}<\right.$ 1) but a comparable amount of high energies, so that the energy histograms have more tenuous but harder tails. This reflects a change of the nature in acceleration from frequent and small energy increments to rare but violent energy gains, in the course of which the stochastic process leaves the domain of attraction of the central limit theorem.

\section{Summary and discussion}

We have simulated gyrokinetic electron orbits in constant- $\alpha$ force-free magnetic fields with anomalous resistivity $\eta$. The latter is localized in (postulated) dissipation regions where the magnetic twist $\nabla \times \hat{\boldsymbol{b}}$ exceeds a given threshold $u_{\mathrm{c}}$, corresponding to a critical scale $1 / u_{\mathrm{c}} \sim 3000 \mathrm{~km}$. The dissipation regions cover about $10^{-3}$ of the simulated slab volume and can be characterized by a column height $h_{\mathrm{d}}$ of 30 to $300 \mathrm{~km}$. The (parallel) dissipative electric field exceeds the Dreicer field by one or two orders of magnitude and yields direct acceleration of runaway electrons. In general, the electrons visit numerous dissipation regions before reaching relativistic energies, and arrive at the chromosphere before escaping to the higher corona. The latter ordering agrees with an observed trend for HXR to precede 

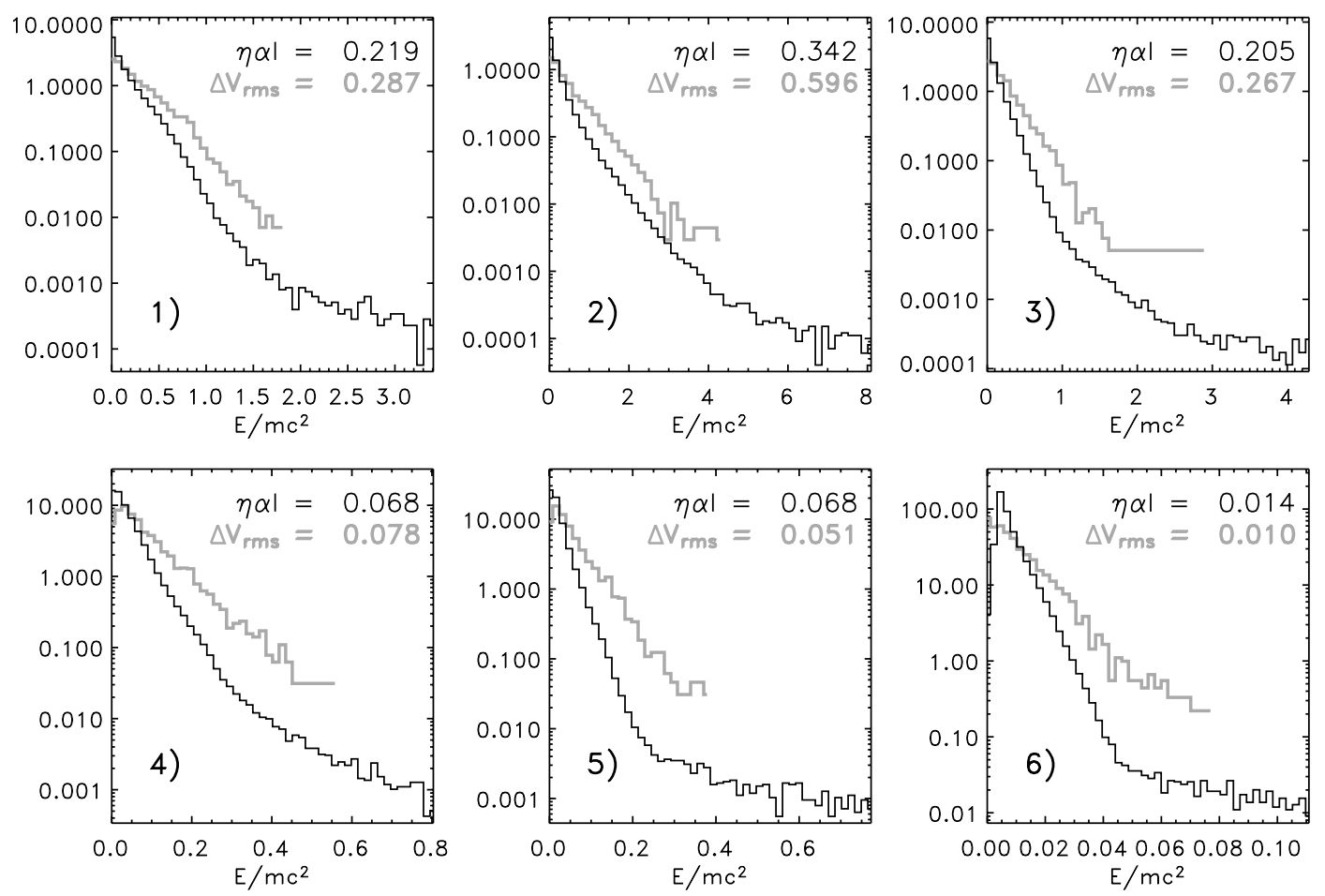

Fig. 13. The distribution of precipitating, escaping, plus trapped particle energy (black line) and the distribution of voltage drops (gray line).

type III bursts (Aschwanden et al. 1992; Arzner \& Benz 2005), also in terms of absolute time delays.

From a physics point of view, one may compare the energies reached in Fig. 12 to the voltage drop along the magnetic field line inside a (simply connected) dissipation region $\mathcal{D}$. (We use here the term "voltage drop" rather than "potential drop" since $\boldsymbol{E}=\eta \boldsymbol{j}$ is not a potential field.) The quantity $\Delta V=\int_{\mathcal{D}} \boldsymbol{E} \cdot \mathrm{d} \boldsymbol{l}$ gives an upper limit to the energy that can be gained inside a single dissipation region; reflection from converging field lines (and also deceleration from $\boldsymbol{E}$ itself) does generally prevent the particles from exploiting the full voltage drop. By choosing $5 \times 10^{3}$ random points inside the dissipation regions and following the magnetic field lines going through these points, we find the distribution of voltage drops as shown in Fig. 13, together with the distribution of all terminal electron energies. Both kinetic energy and voltage drops are measured in units of the electron rest mass. As can be seen, the two distributions scale similarly in energy from one simulation to another (note the different energy axes!), and the sub-exponential tails of the particle energy occur above the largest voltage drop present in the simulation. This agrees with the observation that many dissipation regions are visited before the particles reach the highest energies. The root mean square voltage drops $\Delta V_{\mathrm{rms}}$ are of the order of the voltage drop across the magnetogram resolution, $\eta \alpha l$. The distribution of voltage drops decays slower than the distribution of particle energies, which relates to the fact that $\Delta V_{\mathrm{rms}}$ is merely an upper bound on the kinetic energy gain. The peak of the particle distributions at lowest energies is mostly due to the trapped component.

The present model predicts a statistical preference for the HXR to occur at one magnetic polarity, because of the assumption of constant $\alpha$, by which the electric field $\boldsymbol{E}=\eta \alpha \boldsymbol{B}$ is always parallel $(\alpha>0)$ or antiparallel $(\alpha<0)$ to the magnetic field. The electrons tend to impact the chromosphere not in the centre of sunspots or filaments (where $|\boldsymbol{B}|$ is largest) but at their borders. Ions, due to their opposite charge, should tend to impact at opposed magnetic polarity (but the ion dynamics is not directly comparable to the electron dynamics because of the large mass ratio). The experimental verification or falsification of this model prediction, using non-randomized force-free extrapolations and spatially-resolved X-ray (e.g., RHESSI) observations of flaring active regions, will be the subject of future investigations. It should also be pointed out that the impact map (Fig. 9) is obtained from simultaneous injection in all dissipation regions. If injection were restricted to a single dissipation region or group of dissipation regions, then only few impact regions would occur, as usually observed in individual solar flares.

Finally, we should mention that the present approach has many caveats. Most prominent among them is that test particle simulations cannot tell us anything about the absolute number of electrons that are accelerated, and their application to the real solar corona requires caution in order to match global electrodynamic constraints (i.e. return currents; Spicer \& Sudan 1984). As a rule, our simulations can only account for a tenuous high-energy tail, because the back-action on the electromagnetic field and Coulomb collisions are neglected. Then, we used magnetograms of non-flaring active regions here - where the force-free extrapolation should be a good approximation - and have thus envisaged "quiescent" coronal heating by nanoflares rather than large isolated events. The observable predictions, though, address sizable flares where all types of emission can be detected.

We suggest that the actual flares are generated by a similar process, and should thus have similar characteristics. Conversely, the non-flaring active regions are constantly doing what flaring active regions do but in smaller, possibly undetected numbers. This coronal process may account for spatial $\mathrm{X}$-ray and radio fine structures in quiet solar regions (Benz et al. 1997) and non-thermal electrons measured in space during quiet times ("superhalo"; Lin 1998), as an alternative explanation of MHD wave acceleration in the solar wind. Also, the assumption of a time-independent magnetic field is only an approximation. 
It is certainly violated during large flares with re-structuring of the global magnetic topology. Also, it does not formally account for the continuous footpoint motion driving the Parker (1983) scenario. However, it may be a reasonable approximation for medium-size flares, where the Parker mechanism does not destroy the overall magnetic field structure. This point of view is supported by the work of Aulanier et al. $(2005 \mathrm{a}, \mathrm{b})$ who numerically study the evolution of magnetic flux tubes and quadrupolar configurations under sub-Alfvénic photospheric motion. Their (incompressible, resistive) MHD simulations demonstrate that current sheet formation and topological changes may be described by a sequence of quasi-equilibria, so that a static approximation over a few ten seconds seems appropriate. With regard to particle acceleration, the temporal evolution of the magnetic fields is expected to decrease trapping and facilitate both precipitation and escape.

Acknowledgements. The authors thank Emmanuela Rantsiou and Manolis Georgoulis for help with the SOHO/MDI magnetograms, and Arnold Benz and Gregory Fleishman for helpful discussions and comments. This work was supported in part by the Research Training Network (RTN) "Theory, Observation and Simulation of Turbulence in Space Plasmas", funded by the European Commission (contract No. HPRN-eT-2001-00310).

\section{Appendix A: Numerical implementation}

\section{A.1. Regime switching and accuracy control}

The choice of the orbit integration method, exact or gyrokinetic, depends on two constraints. From a physical point of view, the gyrokinetic approximation requires the Larmor radius to be small compared to the scale of the magnetic field, $\rho \ll l$. From a numerical point of view, the gyrokinetic approximation is faster than exact orbit integration whenever the latter is dominated by particle motion rather than by field evaluation. Indeed, if $\tau_{\mathrm{f}} \mathrm{de}-$ notes the CPU time needed for a single field evaluation and $\tau_{\mathrm{g}}$ is the CPU time needed to resolve a single gyration, then the gyrokinetic approximation is, by a factor

$f=\frac{\tau_{\mathrm{g}}}{\tau_{\mathrm{f}}} \times \frac{l}{\rho}$,

faster than exact orbit tracking. The time $\tau_{\mathrm{g}}$ is approximately independent of the integration scheme as long as this is optimal, and a typical value on a medium-size workstation is found to be $\tau_{\mathrm{g}} \sim 5 \times 10^{-5} \mathrm{~s}$. The time cost of a single field evaluation, on the same hardware, is $\tau_{\mathrm{f}} \sim N_{k} \times 5 \times 10^{-6} \mathrm{~s}$ with $N_{k}$ the number of Fourier components, so that the gyrokinetic method is numerically beneficial for $N_{k}<10 \times l / \rho$. Thus, if the gyrokinetic approximation is physically allowed $(l / \rho \gg 1)$, then it is also numerically beneficial in the sparse-Fourier representation; the upper bound on $N_{k}$ is rarely met in practice.

We turn now to the precise (and implemented) formulation of regime switching and accuracy control. The gyrokinetic approximation requires that the magnetic field change slowly across a Larmor radius, $(\nabla \boldsymbol{B}) \cdot \boldsymbol{\rho}<\epsilon_{\mathrm{g}} B$, where $\nabla \boldsymbol{B}$ is the Jacobian and $\epsilon_{\mathrm{g}} \ll 1$. For practical purposes, we replace this by the stronger and computationally less expensive constraint $\rho^{2} \sum_{i j}\left|\partial_{i} B_{j}\right|^{2}<\epsilon_{\mathrm{g}}^{2} B^{2}$ with $\rho=|\boldsymbol{p}| / B$. The quantity $\rho$ also includes parallel momentum, so that the condition accounts for particle acceleration inside the dissipation regions. In addition to the gyrokinetic approximation error, there are numerical errors from the finite time step and field computation rate. These are controlled by limiting the Cash-Karp position- and momentum errors and by enforcing re-calculation of the electromagnetic fields if $|\Delta x|^{2} \sum_{i j}\left|\partial_{i} B_{j}\right|^{2}>\epsilon_{\mathrm{B}}^{2}$, where $\Delta \boldsymbol{x}=\boldsymbol{x}-\boldsymbol{x}_{\text {old }}$ is the
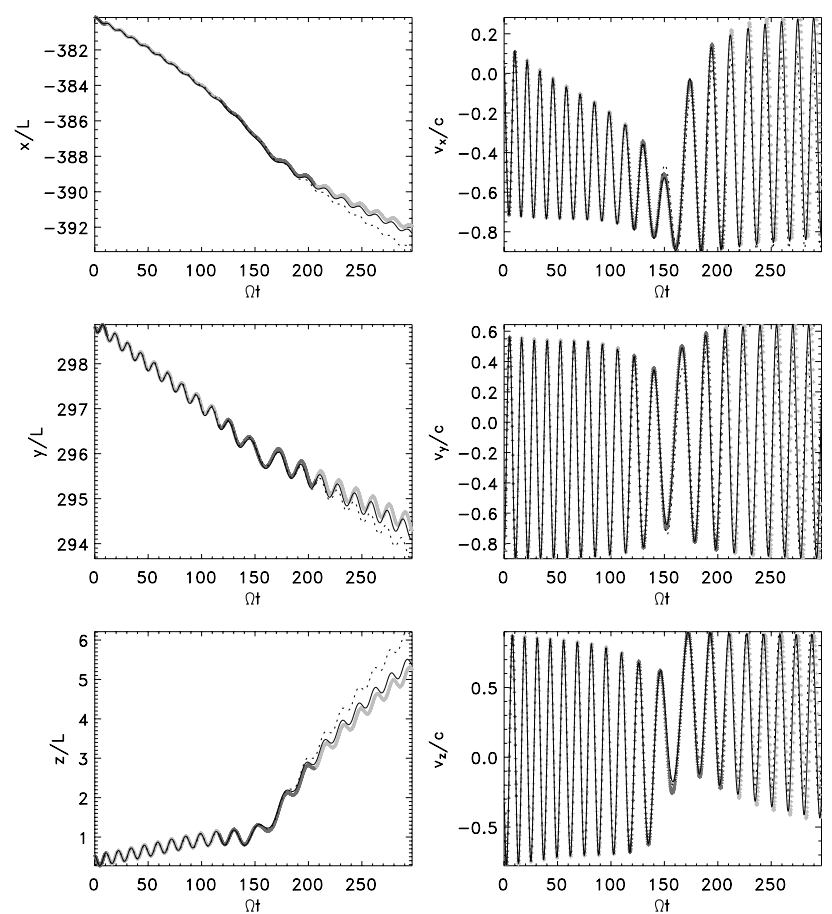

Fig. A.1. Benchmark of the orbit integration schemes, showing position (left column) and velocity (right column) of a relativistic electron in strongly curved $\left(l / l_{0}=10\right)$ magnetic fields. The solid line represents exact orbits (Eqs. (8), (9)); dotted line, gyrokinetic approximation (Eqs. (10)-(14)). Gray crosses represent the hybrid mode, with the exact regime marked as dark and the gyrokinetic regime marked as light.

distance to the last field-evaluation point $\boldsymbol{x}_{\text {old }}$. Using the same Jacobian, the fields are linearly extrapolated from $x_{\text {old }}$. In order to avoid relentless switching between exact and gyrokinetic regimes, a minimum duration of one gyro period in each of the regimes is enforced.

\section{A.2. Benchmarks}

The exact orbits have been computed with different integrationand field evaluation schemes. The time integrators include the Verlet scheme and other leapfrog variants, and Runge-Kutta schemes with and without adaptive time stepping. The field evaluation was done either pointwise or with linear interpolation involving $\nabla \boldsymbol{B}$. Once the exact orbits were established, they were used to benchmark the gyrokinetic orbits. As an example, Fig. A.1 displays individual Cartesian components of position and velocity in an extreme situation, i.e. for a highly relativistic $(v=0.99 c)$ particle with small magnetic scales $\left(l / l_{0}=10\right)$, the gyrokinetic approximation, and the hybrid mode with automatic regime switching. In the hybrid method, the particle starts with exact orbit integration and switches to the gyrokinetic description at $\Omega t \sim 10$. It remains then in the gyrokinetic mode, until at $\Omega t \sim 120$ a region of strong magnetic curvature is encountered, where exact orbit tracing is enforced. At $\Omega t \sim 200$ the orbit switches back to the gyrokinetic method. During the gyrokinetic phase, the magnetic moment is conserved to within $0.1 \%$, but varies during the exact-orbit phase by some $80 \%$. As can be seen, the hybrid orbit is closer to the exact result than the purely gyrokinetic orbit. 


\section{References}

Abramowitz, M., \& Stegun, I. 1970, Handbook of mathematical functions, 7th ed. (New York: Dover Publ. Inc.)

Amari, T., Aly, J. J., Luciani, J. F., Boulmezaoud, T. Z., \& Mikic, Z. 1997, Sol. Phys., 174, 129

Alissandrakis, C. E. 1981, A\&A, 100, 197

Arzner, K., \& Benz, A. 2005, Sol. Phys., 231, 117

Arzner, K., \& Vlahos, L. 2004, ApJ, 605, L69

Arzner, K., Knaepen, B., Carati, D., Denewet, N., \& Vlahos, L. 2006, ApJ, 637, 322

Aschwanden, M. J. 2001, ApJ, 560, 1035

Aschwanden, M., \& Benz, A. O. 1988, ApJ, 332, 447

Aschwanden, M. J., \& Güdel, M. 1992, ApJ, 401, 736

Aschwanden, M. J., Montello, M. L., Dennis, B. R., \& Benz, A. O. 1995, ApJ, 440,394

Aulanier, G., Démoulin, P., \& Grapin, R. 2005a, A\&A, 430, 1067

Aulanier, G., Pariat, E., \& Démoulin, P. 2005b, A\&A, 444, 961

Benz, A. O., Krucker, S., Acton, L. W., \& Bastian, T. 1997, A\&A, 320, 993

Benz, A. O., Grigis, P. C., Csillaghy, A., \& Saint-Hilaire, P. 2005, Sol. Phys., 226,121

Brizard, A. J., \& Chan, A. A. 1999, Phys. Plasmas, 6, 4548

Chiu, Y. T., \& Hilton, H. H. 1977, ApJ, 212, 873

Dmitruk, P., Matthaeus, W. H., Seenu, N., \& Brown, M. R. 2003, ApJ, 597, L81

Dmitruk, P., Mattheus, W. H., \& Seenu, N. 2004, ApJ, 617, 667

Dreicer, H. 1960, Phys. Rev., 117, 329

Dumont, S., Mouradian, Z., Pecker, J.-C., Vial, J.-C., \& Chipman, E. 1982, Sol. Phys., 83, 27

Fleishman, G. D., \& Melnikov, V. F. 1998, Phys. Uspekhi, 41, 1157

Fleishman, G. D., Gary, D. E., \& Nita, G. M. 2003, ApJ, 593, 571

Fleishman, G. D., Nita, G. M., \& Gary, D. E. 2005, ApJ, 620, 506

Fludra, A., Del Zanna, G., Alexander, D., \& Bromage, B. J. I. 1999, J. Geoph. Res., 104, 9709

Gallagher, P. T., Mathioudakis, M., Keenan, F. P., Phillips, K. J. H., \& Tsinganos, K. 1999, ApJ, 524, L133

Gary, G. A. 1989, ApJS, 69, 323

Georgoulis, M., \& Vlahos, L. 1998, A\&A, 336, 721

Giménez de Castro, C. G., Silva, A. V., Trottet, G., et al. 2005, AIP Conf. Proc., 784,566
Grigis, P., \& Benz. A. O. 2004, A\&A, 426, 1093

Güdel, M., Benz, A. O., \& Aschwanden, M. 1991, A\&A, 251, 285

Gudiksen, B. V., \& Nordlund, A. 2002, ApJ, 572, L113

Helander, P., \& Sigmar, D. 2002, Collisional transport in Magnetized Plasmas, Cambridge Monographs on Plasma Physics (Cambridge Univ. Press)

Karimabadi, H., Menyuk, C. R., Sprangle, P., \& Vlahos, L. 1987, ApJ, 316, 462

Koch, H. W., \& Motz, J. W. 1959, Rev. Mod. Phys., 31, 920

Kotov, V. A., Kotov, S. A., \& Setyaev, V. V. 2002, Sol. Phys., 209, 233

Kuijpers, J., \& Slottje, C. 1976, Sol. Phys., 46, 247

Lee, J., \& Gary, D. E. 2000, ApJ, 543, 457

Lin, R. P. 1998, Space Sci. Rev., 86, 61

Littlejohn, R. B. 1981, Phys. Fluids, 24, 1730

Littlejohn, R. B. 1983, J. Plasma Phys., 29, 111

Littlejohn, R. B. 1988, Phys. Rev. A, 38, 6034

MacLeod, M. A. 1995, Inv. Probl., 11, 1087

Matthaeus, W. H., \& Lamkin, S. L. 1986, Phys. Fluids, 29, 2513

Nita, G. M., Gary, D. E., \& Fleishman, G. D. 2005, ApJ, 629, L65

Nobile, A. G., Ricciardi, L. M., \& Sacerdote, L. 1985, J. Appl. Prob., 22, 360

Papadopoulos, K. 1980, in Dynamics of the Magnetosphere, ed. S.-I. Akasofu (Dordrecht: Reidel)

Parker, E. N. 1972, ApJ, 174, 499

Parker, E. N. 1983, ApJ, 264, 635

Parker, E. N. 1993, ApJ, 414, 389

Petrie, G. J. D., \& Lothian, R. M. 2003, A\&A, 398, 287

Press, W., Teukolsky, S., Vetterling, W., \& Flannery, B. 1998, Numerical recipes in C, 2nd ed. (Cambridge: Cambridge Univ. Press)

Rantsiou, E. 2004, Reconstruction of coronal force-free magnetic fields with constant $\alpha$ from photospheric observations, Diploma Thesis, Aristotle University of Thessaloniki, Greece

Saint-Hilaire, P., \& Benz, A. O. 2003, Sol. Phys., 216, 205

Schlickeiser, R. 2003, Springer Lect. Notes Phys., 612, 230

Schott, G. A. 1912, Electromagnetic Radiation, Cambridge

Schumacher, J., \& Kliem, B. 1996, Phys. Plasmas, 3, 4703

Spicer, D. S., \& Sudan, R. N. 1984, ApJ, 280, 448

Török, T., \& Kliem, B. 2001, Proc. 6th Internat. School/Symposium ISSS-6, ed. J. Büchner, Garching, Germany, 364

Vlahos, L., \& Georgoulis, M. 2004, ApJ, 603, L61

Wheatland, M. S. 1999, ApJ, 518, 948 